\title{
O EFEITO DO BAP (6-BENZILAMINOPURINA) SOBRE A INDUÇÃO DE BROTOS EM EXPLANTES DE PAU-BRASIL
}

\author{
Ana Katarina Oliveira Aragão ${ }^{1}$, Magdi Ahmed Ibrahim Aloufa ${ }^{2}$, Igor do Amaral Costa ${ }^{3}$
}

(recebido: 18 de maio de 2010; aceito: 28 de abril de 2011)

RESUMO: A Mata Atlântica brasileira foi intensamente degradada, restando hoje cerca de 7\% a 8\% de sua área original, índices que tornaram crescente a preocupação com a conservação, principalmente, a dos espécimes em extinção. Ao todo, são 276 espécies de árvores e arbustos ameaçados e, dentre esses, a pesquisa em questão optou por estudar alternativas de resguarde ao Pau-Brasil (Caesalpinia echinata Lam.). Nesse sentido, a maioria dos estudos desenvolvidos, ou avalia o efeito das citocininas sobre a indução de calogênese, ou preocupa-se em aperfeiçoar metodologias de criopreservação. Visando a ampliar a gama de conhecimentos em relação às técnicas biotecnológicas que viabilizam a conservação da $C$. echinata, no presente trabalho avaliou-se o efeito do 6-benzilaminopurina (BAP) e do tipo de explante sobre a indução de brotações em Pau-Brasil. Para isso, os explantes foram inoculados em meio MS básico e em MS suplementado com 2,5; 3,5; e 4,5 $\mu \mathrm{M}$ de BAP e mantidos em sala de crescimento, durante 40 dias sob condições controladas de fotoperíodo e temperatura. O delineamento fatorial adotado foi o $2 \times 4$, com três repetições. As variáveis analisadas foram o percentual de brotações, calogênese e oxidações, sendo suas médias comparadas por Tukey a 5\% de probabilidade. Os resultados mostraram que houve influência significativa do BAP apenas na indução de brotações e do tipo de explante nessa e nas demais variáveis. Dessa forma, concluiu-se que, in vitro, o explante do tipo nodal é mais responsivo à ação do BAP e que a concentração de 2,5 $\mu \mathrm{M}$ é a indicada para induzir brotações em Pau-Brasil.

Palavras-chave: Micropropagação in vitro, citocinina, calogênese e oxidações.

\section{EFFECT OF BAP (6-BENZYLAMINOPURINE) ON SHOOT INDUCTION IN EXPLANTS OF BRAZILWOOD}

\begin{abstract}
The Brazilian Atlantic forest has been subjected to intense degradation, with only about $7 \%$ to $8 \%$ of its original area remaining today. This situation has raised concerns over the conservation of species threatened with extinction. In all, 276 tree and bush species are under threat, out of which this study chose to evaluate alternatives for protecting brazilwood 'Pau-Brasil' (Caesalpinia echinata Lam.). Most studies performed so far on this subject either evaluate the effect of cytokinins on induction of callogenesis or focus on improving cryopreservation methodologies. In an attempt to expand knowledge about biotechnological techniques enabling conservation of C. echinata, this work evaluated the effect of 6-benzylaminopurine (BAP) and explant type on induction of shoots in brazilwood. To attain that, explants were inoculated into basic MS medium and into MS medium supplemented with $2.5 \mu \mathrm{M}, 3.5 \mu \mathrm{M}$ and $4.5 \mu \mathrm{M}$ of BAP, and kept in a growth room for 40 days under controlled photoperiod and temperature conditions. A $2 \times 4$ factorial design was adopted, with three replicates. Analyzed variables included shoot percentage, callogenesis and oxidations, and means were compared by the Tukey test at the 5\% probability level. Results showed a significant influence of $B A P$ only on shoot induction, and of explant type on that variable and on other variables too. It was concluded that, under in vitro conditions, the nodal type of explant is more responsive to BAP action and that $2.5 \mu M$ is the recommended concentration for shoot induction in brazilwood.
\end{abstract}

Key words: In vitro micropropagation, cytokinin, callogenesis and oxidations.

\section{INTRODUÇÃO}

A Mata Atlântica é a segunda maior floresta pluvial tropical do continente Americano, perdendo espaço apenas para a Amazônia. Sua principal característica concentra-se nos fatos de apresentar uma alta diversidade de espécies e um elevado grau de endemismo, atributos que, na atualidade, encontram-se fortemente ameaçados.
Segundo Tabarelli et al. (2005), ao longo dos dois últimos séculos, ações como a retirada da cobertura vegetal, a substituição de florestas por áreas de monocultivo e/ ou pastagens, a extração de madeira, as pressões de ocupação e o desmatamento desordenado, contribuíram para a destruição de grande parte do Bioma Atlântico. Tanto que, hoje, restam apenas cerca de $7 \%$ a $8 \%$ da sua área original. E é em decorrência de tão alarmante índice

\footnotetext{
${ }^{1}$ Engenheira Agrônoma, Doutoranda em Desenvolvimento e Meio Ambiente - Departamento de Botânica, Ecologia e Zoologia/DBEZ- Universidade Federal do Rio Grande do Norte - 59072-970 - Natal, RN - katnega@hotmail.com

${ }^{2}$ Engenheiro Agrônomo, Professor Ph.D. em Ciências Biológicas com ênfase em Cultura de Tecidos - Departamento de Botânica, Ecologia e Zoologia/DBEZ - Universidade Federal do Rio Grande do Norte - 59072-970 - Natal, RN - magdi_aloufa@bol.com.br

${ }^{3}$ Biólogo, Estagiário do Laboratório de Biotecnologia Vegetal - Departamento de Botânica, Ecologia e Zoologia/DBEZ - Universidade Federal do Rio Grande do Norte - 59072-970 - Natal, RN - igordoamaralcosta@yahoo.com.br
}

Cerne, Lavras, v. 17, n. 3, p. 339-345, jul./set. 2011 
que vem tornando-se crescente a preocupação com a conservação, principalmente, no que diz respeito aos espécimes categorizados como propícias a extinção.

De acordo com Brasil (2008), 472 espécies vegetais em todo o Brasil encontram-se ameaçadas de extinção, sendo 276 nativas da Mata Atlântica. O número de profissionais envolvidos, os recursos e o tempo necessários para estudar simultaneamente métodos científicos de resguarde a essa quantidade de espécimes dificultariam em sobremaneira a execução de uma pesquisa. Para contornar essas restrições, o indicado é empregar uma metodologia e observar sua influência sobre o menor número possível de espécies (GRATTAPAGLIA; MACHADO, 1998). Assim, as investigações ficam mais concentradas, conferindo maior exequibilidade ao estudo.

Das árvores e arbustos em risco na Floresta Atlântica, a pesquisa em questão elegeu como objeto de estudo a Caesalpinia echinata Lam., vulgarmente conhecida como Pau-Brasil. A opção deveu-se ao fato de ainda existirem lacunas no conhecimento referentes à multiplicação in vitro desse exemplar. Conforme Torres et al. (1999), a maioria das pesquisas desenvolvidas com a C. echinata ou avaliam o efeito das citocininas sobre a indução de calogênese ou preocupam-se em aperfeiçoar métodos e técnicas de criopreservação.

O Pau-Brasil (Leguminosae - Caesalpinoideae), segundo Barbedo et al. (2002), tem um ótimo potencial ornamental em razão da sua beleza e raridade, apresentando porte elegante, copa arredondada, folhas verde-brilhantes e flores em cacho amarelo-ouro. Sua madeira é pesada, dura e contém cerne de coloração vermelha quando recémcortada. O status de extinção decorreu de sucessivos anos de exploração indiscriminada e foi intensificado pela supressão do Bioma Atlântico, evoluindo a proporções que, na atualidade, remetem sérias preocupações com sua conservação (ROCHA, 2004).

Nas últimas décadas, o emprego da cultura de tecidos tem assegurado com eficiência a sobrevivência dos espécimes florestais (GUERRA et al., 1999). Nesse sentido, os procedimentos mais usuais são: a micropropagação in vitro, a microenxertia, a conservação de germoplasma e a cultura de embriões, ovários ou anteras. A aplicação de cada uma dessas metodologias depende, dentre outros fatores, da disponibilidade do material para ser trabalhado e dos recursos designados à pesquisa (GUERRA; NODARI, 2006). No caso da Caesalpinia echinata Lam., em virtude da restrita disponibilidade de semente e do fato das mesmas apresentarem baixa viabilidade (de 30 a 90 dias em condições naturais), a técnica mais apropriada é a micropropagação in vitro (ENDRES et al., 2006).

A propagação vegetativa in vitro ou micropropagação constitui uma excelente via de conservação para espécies como o Pau-Brasil, pois contorna as condições que comprometem a reprodução natural da planta e promove a multiplicação em grande escala dos exemplares, podendo os novos indivíduos serem utilizados em programas de reflorestamento e em projetos de recomposição de áreas degradadas (MONDO et al., 2008).

Apoiada nas prerrogativas de que poucos são os estudos sobre a micropropagação do Pau-Brasil; de que esse vegetal apresenta limitações fisiológicas naturais que tornam a adoção da semente como explante pouco funcional; de que as citocininas são reguladores de crescimento ideais para estimular o desenvolvimento de gemas axilares (GALVANESSE et al., 2007); e de que, para tornar continua as demais fases de propagação vegetativa in vitro, é preciso gerar o maior número possível de brotos, nesta pesquisa, objetivou-se avaliar o efeito do BAP e do tipo de explante sobre a indução de brotações em Pau-Brasil.

Dentre as citocininas que servem a essa finalidade, a 6-Benzilaminopurina (BAP) foi eleita, porque, dentro dessa classe, é o fitorregulador natural que tem se mostrado mais eficaz na multiplicação de diversas espécies lenhosas, além disso, ainda proporciona o menor custo de aquisição. A ação desse regulador de crescimento pode ser justificada pela capacidade que os tecidos vegetais têm de metabolizar os hormônios naturais mais rapidamente do que os sintéticos (BORGES JÚNIOR et al., 2004).

\section{MATERIAL E MÉTODOS}

O ensaio foi realizado no Laboratório de Biotecnologia Vegetal do Departamento de Botânica, Ecologia e Zoologia da Universidade Federal Rio do Grande do Norte - UFRN, em Natal-RN, no período de agosto a outubro de 2008.

A primeira etapa desse experimento constou da seleção das fontes de explante. Do banco de matrizes ex vitro, conservado na casa de vegetação do Centro de Biociências da UFRN, escolheram-se as plantas de PauBrasil que apresentavam melhor padrão fitossanitário (vigor e ausência de pragas e doenças) e delas removeram-se hastes não-lenhosas com sete centímetros de comprimento. De cada matriz foi seccionada uma única haste. 
No laboratório, em capela de fluxo laminar, essas porções de caule foram desinfestadas com banho de três minutos em álcool 70\%, seguido por imersão de 10 minutos em hipoclorito de sódio na concentração de $2 \% \mathrm{e}$ finalizado com três submersões sucessivas de 15 minutos (cada) em água destilada e autoclavada.

Concluída a desinfecção, retirou-se de cada haste um segmento nodal e outro internodal, ambos com $1,5 \mathrm{~cm}$ de comprimento. Esses, por sua vez, foram individualmente inoculados, na posição vertical, em frascos de vidro (previamente esterilizados), contendo $30 \mathrm{~mL}$ dos seguintes meios de cultura: MS básico; MS acrescido de 2,5 $\mu \mathrm{M}$ de BAP; MS adicionado de 3,5 $\mu \mathrm{M}$ de BAP; e MS mais 4,5 $\mu \mathrm{M}$ de BAP.

Depois de inoculados, os explantes foram mantidos em sala de crescimento onde permaneceram durante 40 dias sob condições controladas de temperatura $\left( \pm 26^{\circ} \mathrm{C}\right)$ e luminosidade (16 h. luz/dia).

$\mathrm{O}$ delineamento experimental adotado foi o fatorial 2x4 (2 tipos de segmento x 4 concentrações de 6-benzilaminopurina), com três repetições. Por repetição, os tratamentos continham 10 unidades experimentais. Cada unidade experimental era composta por um único explante (nodal ou internodal), inoculado em meio MS básico acrescido, ou não, de única dose do fitorregulador.

Observaram-se como variáveis: a indução de brotações; a formação de calos; e a ocorrência de oxidação. As avaliações foram quanto ao número de brotos; calos e oxidações que surgiram em cada unidade experimental.

Os dados coletados foram percentualizados e submetidos à análise da variância. As médias encontradas foram comparadas estatisticamente pelo teste de Tukey ao nível de $5 \%$ de probabilidade.

\section{RESULTADOS E DISCUSSÃO}

A indução de brotos em Pau-Brasil foi influenciada significativamente pelo tipo de explante, sendo os segmentos nodais os mais propícios ao desenvolvimento dos brotos (Tabela 1). Nessas condições, observou-se ainda que a ação da 6-benzilaminopurina também foi condicionada ao explante utilizado, pois, houveram brotações apenas nos segmentos nodais cultivados em meio MS adicionado de BAP. Quando cultivados em meio MS básico sem o regulador de crescimento os segmentos nodais não apresentaram quaisquer reações.

Em relação aos segmentos internodais, apesar de terem sido cultivados sobre as mesmas condições dos nodais, os resultados apresentaram-se diferentes: nos internódios não surgiram brotações, e, por conseguinte, a ação do BAP não foi efetiva.

Tabela 1 - Influência do tipo de explante em meio MS com diferentes de 6-benzilaminopurina sobre a indução de brotos em Caesalpinia echinata Lam. depois de 40 dias de cultivo.

Table 1 - Influence of explant type in MS medium with different 6-benzylaminopurine concentrations on shoot induction in Caesalpinia echinata Lam. after 40 days of cultivation.

\begin{tabular}{ccc}
\hline \multirow{2}{*}{$\begin{array}{c}\text { Concentrações de } \\
\text { BAP }(\mu \mathrm{M})\end{array}$} & \multicolumn{2}{c}{ Tipo de Explante/Resposta } \\
\cline { 2 - 3 } & $\begin{array}{c}\text { Nodal } \\
\text { Indução de } \\
\text { Brotações }(\%)\end{array}$ & $\begin{array}{c}\text { Internodal } \\
\text { Indução de } \\
\text { Brotações }(\%)\end{array}$ \\
\hline 0,0 & $0,00 \mathrm{Aa}$ & $0,00 \mathrm{Aa}$ \\
2,5 & $31,33 \mathrm{Ba}$ & $0,00 \mathrm{Aa}$ \\
3,5 & $20,00 \mathrm{Ba}$ & $0,00 \mathrm{Aa}$ \\
4,5 & $34,65 \mathrm{Ba}$ & $0,00 \mathrm{Aa}$ \\
\hline
\end{tabular}

Médias seguidas de letras minúsculas diferentes na mesma linha diferem entre si a $5 \%$ de probabilidade, enquanto as seguidas de mesmas letras maiúsculas em uma única coluna não diferem entre si ao mesmo nível de probabilidade, ambas avaliadas pelo teste de Tukey.

A interação entre BAP e tipo de explante também foi observada na Tabela 2, onde a formação de calos ocorreu apenas nos segmentos nodais cujo meio de cultivo foi suplementado com 6-benzilaminopurina.

Tabela 2 - Efeito de diferentes concentrações de BAP (6-benzilaminopurina) sobre a calogênese em segmentos nodais e internodais de Pau-Brasil.

Table 2 - Influence of different BAP (6-benzylaminopurine) concentrations on callogenesis in nodal and internodal segments of brazilwood.

\begin{tabular}{ccc}
\hline \multirow{2}{*}{$\begin{array}{c}\text { Concentrações de } \\
\text { BAP }(\mu \mathrm{M})\end{array}$} & \multicolumn{2}{c}{ Tipo de Explante/Resposta } \\
\cline { 2 - 3 } & $\begin{array}{c}\text { Nodal } \\
\text { Formação de } \\
\text { Calos }(\%)\end{array}$ & $\begin{array}{c}\text { Internodal } \\
\text { Formação de } \\
\text { Calos }(\%)\end{array}$ \\
\hline 0,0 & $00,00 \mathrm{Aa}$ & $0,0 \mathrm{Ab}$ \\
2,5 & $25,00 \mathrm{Ba}$ & $0,0 \mathrm{Ab}$ \\
3,5 & $28,33 \mathrm{Ba}$ & $0,0 \mathrm{Ab}$ \\
4,5 & $29,67 \mathrm{Ba}$ & $0,0 \mathrm{Ab}$ \\
\hline
\end{tabular}

Médias seguidas de letras minúsculas diferentes na mesma linha diferem entre si a $5 \%$ de probabilidade, enquanto as seguidas de mesmas letras maiúsculas em uma única coluna não diferem entre si ao mesmo nível de probabilidade, ambas avaliadas pelo teste de Tukey.

Cerne, Lavras, v. 17, n. 3, p. 339-345, jul./set. 2011 
De acordo com Vieira e Monteiro (2002), as citocininas estimulam o desenvolvimento de porções de tecido meristemático, mais especificamente das gemas axilares, desencadeando o crescimento e divisões celulares que culminam ou no surgimento de uma brotação, ou na formação de calos. Brunette et al. (2006) complementam, afirmando que o balanço entre as quantidades de citocininas exógenas e auxinas endógenas varia conforme o tecido utilizado como explante.

No caso das Tabelas 1 e 2, nos segmentos internodais não foi detectada, respectivamente, a indução de brotações e a calogênese. Sousa et al. (2007), explicam que a inativação das porções meristemáticas muitas vezes acontece porque a competência do explante em assimilar os estímulos provocados pela classe hormonal não alcança a razão necessária para induzir os processos organogenéticos.

Segundo Guerra e Noradi (2006), para que brotos sejam originados, é necessário que no desfecho do balanço auxina $\mathrm{x}$ citocininas as concentrações de citocininas se sobressaiam; para ocorrer a calogênese a condição é haver um equilíbrio no balanço hormonal. Dessa forma, os resultados encontrados nesta pesquisa são justificados. Aqui, os brotos surgiram nos segmentos nodais porque esse tipo de explante mostrou-se mais propício ao sobressalto das citocininas, havendo maior expressão quando foi adicionada a dosagem de $4,5 \mu \mathrm{M}$ de BAP.

As pesquisas de Mantovani et al. (2007) confirmaram a eficiência do BAP como indutor de brotações em segmentos nodais de Cordia trichotoma (Vellozo) Arrabida ex Steudel. Em comparação com o TDZ, o estudo desse autor apontou que a ação da 6-benzilaminopurina induziu a um maior número de brotos. $\mathrm{O}$ mesmo aconteceu no trabalho de Ribas et al. (2005) com Peroba-Rosa (Aspidosperma polyneuron), onde as brotações aumentaram expressivamente a partir da adição de $4,5 \mu \mathrm{M}$ de BAP.

Outros autores como Coelho (1999), Fermino Júnior et al. (2009), Fráguas et al. (2004), Ponte (1999), Rocha et al. (2007) e Schottz et al. (2007), ao estudarem, respectivamente, o efeito do BAP em Teca (Tectona grandis L.f), Eucalipto (Eucalyptus globulus Labill), Sucupira Branca [Pterodon pubescens (Benth.) Benth.], Mogno (Swietenia macrophylla), Cedro-Canjerana (Cabralea canjerana) e Ficus (Ficus carica), reafirmaram que a presença do BAP, no meio de cultivo, estimulava de forma significativa a produção de brotos em espécies lenhosas.

Como se pôde observar na Tabela 2, a adição de $4,5 \mu \mathrm{M}$ de BAP ao meio nutritivo também foi responsável pela formação do maior percentual de calos $(29,67 \%)$.
Conforme Werner et al. (2009), esse fenômeno foi possibilitado porque explantes juvenis de Caesalpinia echinata Lam. apresentam maiores concentrações endógenas de auxina e essas, quando interagem com as altas concentrações de 6-benzilaminopurina, tendem a manter um equilíbrio. Condição semelhante foi verificada nas pesquisas de Cordeiro et al. (2004) com Paricá (Schizolobium amazonicum Huber ex Ducke) e Soares (2003) com Ingazeiro (Inga vera Subsp. affinis).

Para Pierik (1990), explantes juvenis nãolignificados são mais propícios à calogênese. Todavia, apesar de atenderem a tais requisitos, os segmentos internodais de Pau-Brasil não formaram calos porque oxidaram antes mesmo de concluírem o balanço hormonal (WERNER et al., 2007). Tanto a calogênese quanto a indução de brotações em C. echinata aconteceram do sétimo ao décimo oitavo dia após a inoculação, enquanto que as oxidações ocorreram de terceiro ao décimo dia.

Segundo Teixeira (2001), a oxidação varia de acordo com o tipo de explante. Nesse caso, a oxidação sofreu influência significativa do tipo de explante, sendo os internódios mais propensos às oxidações (Tabela 3).

Tabela 3 - Influência de diferentes concentrações de BAP em dois tipos de explantes quanto às oxidações fenólicas em $C$. echinata Lam.

Table 3 - Influence of different BAP concentrations in two explant types on phenolic oxidations in C. echinata Lam.

\begin{tabular}{ccc}
\hline \multirow{2}{*}{$\begin{array}{c}\text { Concentrações de } \\
\text { BAP }(\mu \mathrm{M})\end{array}$} & \multicolumn{2}{c}{ Tipo de Explante/Resposta } \\
\cline { 2 - 3 } & $\begin{array}{c}\text { Nodal } \\
\text { Ocorrência de } \\
\text { Oxidações }(\%)\end{array}$ & $\begin{array}{c}\text { Internodal } \\
\text { Ocorrência de } \\
\text { Oxidações }(\%)\end{array}$ \\
\hline 0,0 & $10,33 \mathrm{Aa}$ & $80,33 \mathrm{Ab}$ \\
2,5 & $13,67 \mathrm{Aa}$ & $80,33 \mathrm{Ab}$ \\
3,5 & $20,33 \mathrm{Aa}$ & $67,00 \mathrm{Ab}$ \\
4,5 & $13,67 \mathrm{Aa}$ & $80,33 \mathrm{Ab}$ \\
\hline
\end{tabular}

Médias seguidas de letras minúsculas diferentes na mesma linha diferem entre si a $5 \%$ de probabilidade, enquanto as seguidas de mesmas letras maiúsculas em uma única coluna não diferem entre si ao mesmo nível de probabilidade, ambas avaliadas pelo teste de Tukey.

Explantes jovens, na maioria das vezes, minimizam as chances de haver oxidação (CARVALHO et al., 2006). Isso porque os fenóis precursores da síntese de lignina estão presentes em menor quantidade em explantes juvenis (MONTEIRO et al., 2004). Apesar dos segmentos nodais e

Cerne, Lavras, v. 17, n. 3, p. 339-345, jul./set. 2011 
internodais utilizados nesta pesquisa terem sido extraídos de matrizes com a mesma idade (três meses), os internós encontravam-se mais maduros, ou seja, concentravam maiores quantidades de compostos fenólicos que os segmentos nodais e, por esse motivo, oxidaram com maior frequência.

As oxidações fenólicas constituem uma das principais causas de insucesso na propagação vegetativa in vitro em espécies lenhosas. Ao oxidar o explante atravessa um gradativo processo de escurecimento, tem sua absorção de nutriente dificultada e a composição do meio de cultivo é modificada (FICK, 2007). Como medida preventiva podese: adicionar antioxidantes ao meio de cultivo; manter os explantes no escuro durante seu estabelecimento; lavar os explantes em água corrente antes de iniciar o processo de micropropagação in vitro; e utilizar meios de cultura básicos mais diluídos (AUGUSTO, 2001; SATO et al., 2001). Uma última recomendação é ajustar a concentração de hipoclorito de sódio para a porção de tecido com a qual vai se trabalhar. Conforme Almeida et al. (2008), quando a dosagem do desinfestante não é adequada, o explante é lesionado. Essa lesão, por sua vez, aumenta as chances de haver oxidação.

Por fim, ao observarem-se os resultados dos parâmetros avaliados como um todo, percebeu-se que a concentração de 4,5 $\mu \mathrm{M}$ de 6-benzilaminopurina apresenta os maiores percentuais de brotações e calogênese. Já em relação à oxidação, os valores dessa dosagem se igualam aos de 2,5 $\mu \mathrm{M}$. Ainda sobre a analise do contexto geral, percebeu-se que, em se tratando dessas duas concentrações, os valores para indução de brotações $(31,33 \%$ e $34,65 \%$, respectivamente.) e formação de calos $(25,00 \%$ e $29,67 \%$, respectivamente.) apresentaram-se muito próximos, fato que pode gerar certa dúvida no momento de optar pela dosagem com a qual se vai trabalhar. De acordo com Mantell et al. (1994), o sucesso da micropropagação realizada a partir de explantes é condicionado ao desenvolvimento de gemas axilares que originem o maior número de brotos possíveis perante a menor ocorrência de oxidações e calogênese. Considerando essa constatação e visto que a maior formação de calos deu-se nos meios MS adicionados de 4,5 $\mu \mathrm{M}$ de BAP, a concentração de $2,5 \mu \mathrm{M}$ de 6-benzilaminopurina destacou-se junto a propagação in vitro do Pau-Brasil.

\section{CONCLUSÕES}

Os resultados da presente pesquisa evidenciaram que o tipo de explante influencia significativamente nas respostas in vitro e que a ação de BAP sobre a indução de brotos e calogênese somente ocorre em segmentos nodais. Em relação às oxidações há maior expressão nos internódios, sem influência significativa da 6-benzilaminipurina sobre o processo.

Diante do exposto, concluiu-se que, in vitro, os segmentos nodais são os mais propícios à ação do BAP e que a concentração de $2,5 \mu \mathrm{M}$ é a mais indicada para induzir brotações em Pau-Brasil.

\section{REFERÊNCIAS}

ALMEIDA, J. R.; MARTINS, C. R.; DUTRA, L. F. Desinfestação de segmentos nodais de Eucalyptus dunnii visando estabelecimento in vitro. Revista da Faculdade de Zootecnia, Veterinária e Agronomia, Uruguaiana, v. 15, n. 1, p. 54-60, 2008.

AUGUSTO, C. S. S. Micropropagação da amoreirapreta cv. brazos. 2001. 116 p. Dissertação (Mestrado em Agronomia) - Universidade Federal do Paraná, Curitiba, 2001.

BARBEDO, C. J.; BILIA, D. A. C.; FIGUEIREDO, R. de C. L. Tolerância à dessecação e armazenamento de sementes de Caesalpinia echinata Lam. (pau-brasil), espécie da Mata Atlântica. Revista Brasileira de Botânica, São Paulo, v. 25, n. 4, p. 431-439, 2002.

BORGES JÚNIOR, N.; SOBORSA, R. C.; CODER, M. P. M. Multiplicação in vitro de gemas axilares de acácia-negra (Acacia mearnsii De Wild.). Revista Árvore, Viçosa, v. 28, n. 4, p. 751-754, jul./ago. 2004.

BRASIL. Ministério do Meio Ambiente. Lista oficial das espécies da flora brasileira ameaçadas de extinção. Brasília, 2008.

BRUNETTE, J. M. F. C.; OTONI, W. C.; PINHEIRO, A. L.; FONSECA, E. de P. Calogênese in vitro em segmentos de epicótilo de mogno (Swietenia macrophylla King) com uso de 6-benzilaminopurina e ácido $\alpha$-naftalenoacético. Scientia Forestalis, Piracicaba, n. 71, p. 19-24, ago. 2006.

CARVALHO, J. M. F. C.; SILVA, M. M. de A.; MEDEIROS, M. J. L. Fatores inerentes à micropropagação. Campina Grande: Embrapa Algodão, 2006. 28 p.

COELHO, M. C. F. Germinação de sementes e propagação in vitro de sucupira-branca (Pterodon pubescens Benth.). 1999. 119 p. Dissertação (Mestrado em Fitotecnia) -

Universidade Federal de Lavras, Lavras, 1999.

Cerne, Lavras, v. 17, n. 3, p. 339-345, jul./set. 2011 
CORDEIRO, I. M. C. C.; LAMEIRA, O. A.; OHASHI, S. T.; ROSA, L. F. Efeito de BAP sobre a proliferação de brotos in vitro de Schizolobium amazonicum Huber ex Ducke (paricá). Cerne, Lavras, v. 10, n. 1, p. 118-124, jan./jun. 2004.

ENDRES, L.; MARROQUIM, P. M. G.; SANTOS, C. M. dos; SOUZA, N. N. F. Enraizamento de estacas de Pau-Brasil (Caesalpinia echinata Lam.) tratadas com ácido indol butírico e ácido naftaleno acético. Ciência Rural, Santa Maria, v. 37, n. 3, p. 886-889, maio/jun. 2007.

FERMINO JÚNIOR, P. C. P.; NAGAO, E. O.; PEREIRA, J. E. S. Estabelecimento, germinação e multiplicação in vitro de teca (Tectona grandis L.f.) a partir de genótipos da Amazônia Sul-Ocidental. Scientia Forestalis, Piracicaba, v. 37, n. 84, p. 427-435, dez. 2009.

FICK, T. A. Estabelecimento in vitro e propagação de Cordia trichotoma (Vell.) Arrabida ex Steudel (LOURO-PARDO). 2007. 61 p. Dissertação (Mestrado em Engenharia Florestal) Universidade Federal de Santa Maria, Santa Maria, 2007.

FRÁGUAS, C. B.; PASQUAL, M.; PEREIRA, A. R. Multiplicação in vitro de Ficus carica L.: efeito da cinetina e do ácido giberélico. Ciência e Agrotecnologia, Lavras, v. 28, n. 1, p. 49-55, jan./fev. 2004.

GALVANESSE, M. S.; TAVARES, A. R.; AGUIAR, A. F. F. A.; KANASHIRO, S.; CHU, E. P.; STANCATO, G. C.; HARDER, I. C. F. Efeito de ANA, 6-BA e ágar na propagação in vitro de Aechmea blanchetiana (Baker) L.B. Smith, Bromélia nativa da mata atlântica. Revista Ceres, Viçosa, v. 54, p. 63-67, 2007.

GRATTAPAGLIA, D.; MACHADO, M. A. Micropropagação. In: TORRES, A. C.; CALDAS, L. S. (Eds.). Técnicas e aplicações da cultura de tecidos de plantas. Brasília: Embrapa/CNPH, 1998. p. 99-169.

GUERRA, M. P.; NODARI, R. O. Apostila de biotecnologia. Florianópolis: Steinmacher, 2006. 41 p.

GUERRA, M. P.; TORRES, A. C.; TEIXEIRA, J. B. Embriogênese somática e sementes sintéticas. In: TORRES, A. C.; CALDAS, L. S.; BUSO, J. A. (Eds.). Cultura de tecidos e transformação genética de plantas. Brasília: Embrapa/ CBAB, 1999. v. 2.

MANTELL, S. H.; MATTHEWS, J. A.; MCKEE, R. A. Princípios da biotecnologia em plantas: uma introdução à engenharia genética em plantas. Ribeirão Preto: Sociedade Brasileira de Genética, 1994. 344 p.

MANTOVANI, N. C.; FRANCO, E. T. H.; VESTENA, S. Regeneração in vitro de Louro-Pardo (Cordia trichotoma (Vellozo) Arrabida ex Steudel). Ciência Florestal, Santa Maria, v. 11, n. 2, p. 93-101, 2007.

MONDO, V. H. V.; BRANCALION, P. H. S.; CICERO, S. M.; NOVEMBRE, A. D. L. C.; DOURADO NETO, D. Teste de germinação de sementes de Parapiptadenia rigida (Benth.) Brenan (Fabaceae). Revista Brasileira de Sementes, São Paulo, v. 30, n. 2, p. 177-183, 2008.

MONTEIRO, M. B. de O.; PEREIRA, R. P. W.; ABREU, H. dos $\mathrm{S}$. Bioquímica da lignificação de células xilemáticas. Floresta e Ambiente, Seropédica, v. 11, n. 2, p. 48-57, ago./dez. 2004.

PIERIK, R. L. M. Cultivo in vitro de las plantas superiores. Madrid: Mundi, 1990. 326 p.

PONTE, E. M. D. Micropropagação de Eucalyptus globulus sp. Globulus Labill. 1999. 47 p. Dissertação (Mestrado em Produção Vegetal) - Universidade Federal de Pelotas, Pelotas, 1999.

RIBAS, L. L. F.; ZANETTE, F.; KULCHETSKI, L.; GUERRA, M. P. Micropropagação de Aspidosperma polyneuron (Perobarosa) a partir de segmentos nodais de mudas juvenis. Revista Árvore, Viçosa, v. 29, n. 4, p. 517-524, 2005.

ROCHA, S. C.; QUORIN, M.; RIBAS, L. L. P.; KOEHLER, H. S. Micropropagação de Cabralea canjerana. Revista Árvore, Viçosa, v. 31, n. 1, p. 43-50, 2007.

ROCHA, Y. T. Conservação do pau-brasil (Caesalpinia echinata Lam. Leguminosae). In: CONGRESSO DA SOCIEDADE BOTÂNICA DE SÃO PAULO, 15., 2004, Ubatuba. Anais... Ubatuba: Sociedade Botânica de São Paulo, 2004. CD-ROM.

SATO, Y. A.; DIAS, E. C. T.; ANDRADE, L. A. de; SOUZA, V. C. Micropropagação de Celtis sp.: controle da contaminação e oxidação. Cerne, Lavras, v. 7, n. 2, p. 117 123, jul./ago. 2001.

SCHOTTZ, E. S.; KALIL FILHO, A. N.; TRACZ, A. L.; KOEHLER, H. S.; RIBAS, L. L. F.; QUOIRIN, M. Multiplicação in vitro de Swietenia macrophylla King (Meliaceae) a partir de material juvenil. Ciência Florestal, Santa Maria, v. 17, n. 2, p. 109-117, abr./jun. 2007.

Cerne, Lavras, v. 17, n. 3, p. 339-345, jul./set. 2011 
SOARES, G. de A. Aspectos do cultivo in vitro do ingazeiro [Inga vera Willd. subsp. affinis (DC) T. D. Penn]. 2003. 90 p. Dissertação (Mestrado em Fisiologia Vegetal) Universidade Federal de Lavras, Lavras, 2003.

SOUSA, C. da S.; MOREIRA, M. J. S.; BASTOS, L. P.; COSTA, M. A. P. de C.; ROCHA, M. A. C. da; HANSEN, D. S. Germinação e indução de brotações in vitro utilizando diferentes reguladores vegetais em mangabeira (Hancornia speciosa). Revista Brasileira de Biociências, Porto Alegre, v. 5, n. 2, p. 276-278, jul. 2007. Suplemento.

TABARELLI, M.; PINTO, L. P.; SILVA, J. M. C.; HIROTA, M. M.; BRDÊ, L. P. Desafios e oportunidades para a conservação da biodiversidade da Mata Atlântica Brasileira. Megadiversidade, Belo Horizonte, v. 1, n. 1, p. 132-138, jul. 2005.

TEIXEIRA, J. B. Limitações ao processo de cultivo in vitro de espécies lenhosas. Brasília: Embrapa Recursos Genéticos e Biotecnologia, 2001.
TORRES, R. A. A.; MENDANHA, A. B. L.;

BLUMENSCHEIN, A. Estabelecimento de um protocolo para micropropagação de pau-brasil (Caesalpinia echinata Lam.) por cultura de tecidos. In: CONGRESSO NACIONAL DE GENÉTICA, 45., 1999, Gramado. Anais... Gramado: Sociedade Brasileira de Genética, 1999. p. 491-491.

VIEIRA, E. L.; MONTEIRO, C. A. Hormônios vegetais. In: CASTRO, P. R. C. e; SENA, J. O. A. de; KLUGE, R. A. Introdução a fisiologia do desenvolvimento vegetal. Maringá: EDUEM, 2002. p. 79-104.

WERNER, E. T.; CUZZUOL, G. R. F.; PESSOTTI, K. V.; LOPES, F. P.; ROGER, J. A. Controle da calogênese de paubrasil in vitro. Revista Árvore, Viçosa, v. 33, n. 6, p. 987-996, nov./dez. 2009.

WERNER, E. T.; CUZZUOL, G. R. F.; PESSOTTI, K. V.; LOPES, F. P.; ROGER, J. A. Indução de Caesalpinia echinata Lam. (pau-brasil) in vitro. Revista Brasileira de Biociências, Porto Alegre, v. 5, n. 2, p. 1053-1055, jul. 2007. Suplemento.

Cerne, Lavras, v. 17, n. 3, p. 339-345, jul./set. 2011 